\title{
Energy Efficient Communications over the AWGN Relay Channel
}

\author{
Jesús Gómez-Vilardebó, Student Member, IEEE, Ana I. Pérez-Neira, Senior Member, IEEE, \\ and Montse Nájar, Member, IEEE
}

\begin{abstract}
This paper addresses the energy efficiency analysis of the relay channel under additive white Gaussian noise. We consider the rate bounds given by decode and forward and the cut set bound and assume that resources are optimally allocated to maximize the spectral efficiency according to the channel information and the sum network energy. The low energy analysis tools are used to compute the maximum rate per energy (RPE) and the slope of the spectral efficiency as a function of the energy per bit. Using these metrics, the energy efficiency benefit of several capabilities at terminals is investigated. Specifically, we take into account: $i$ ) the phase synchronization between transmitters, ii) the full duplex capability at the relay and iii) the channel access via superposition.
\end{abstract}

Index Terms-Relay channel, energy efficiency, low power communication, wideband regime, receiver constraints, half duplex, synchronism.

\section{INTRODUCTION}

$\mathbf{I}$ $\mathrm{N}$ wireless networks, user cooperation has shown to increase data rates and to provide energy savings [1]. However, these benefits are at the cost of more complex terminals with additional capabilities, such as (see Table I): the $i$ ) phase synchronization between transmitters, that allows terminals to transmit signals that add coherently at the destination; ii) the full duplex (FD) mode at the relay, that allows the relay to receive and transmit simultaneously in the same band; and iii) the channel access via superposition, that jointly with a receiver able to cope with inter-user interference, allows simultaneous transmissions. If FD operation is not possible, the relay works in half duplex (HD) mode and the transmission and reception channels are orthogonal, e.g. time-division. Likewise, if superposition channel access is not possible, then the channels access is orthogonal (OT).

In this letter, we explore the benefits associated with these capabilities by studying the energy efficient regime of the relay channel (RC), where a source communicates to a destination assisted by a dedicated relay. We study the spectral efficiency $\mathrm{R}\left(\frac{E_{b}}{N_{0}}\right)$ as a function of the transmitted energy per information bit relative to the noise spectral level $\frac{E_{b}}{N_{0}}$. In the low power regime there are two metrics of special interest [2]: the maximum rate per energy (RPE) denoted as $\eta$ or, equivalently, the minimum energy per bit $\frac{E_{b}}{N_{0}}$ min and the slope

Manuscript received August 5, 2008; revised April 30, 2009 and July 20, 2009; accepted September 25, 2009. The associate editor coordinating the review of this letter and approving it for publication was $\mathrm{H}$. Dai.

J. Gomez-Vilardebo is with the Centre Tecnologic de Catalunya (CTTC), Castelldefels (Barcelona) Spain (e-mail: jesus.gomez@cttc.es).

A. Perez-Neira and M. Nájar are with the Department of Signal Theory and Communications, (TSC), Universitat Politècnica de Catalunya, (UPC), Jordi Girona, 1-3, 08034 Barcelona, Spain.

This work was partially supported by the Catalan Government under grant SGR2005-00996; by the Spanish Government under projects TEC200508122-C03 and European project ICT-2007.1.1 NEWCOM++ 216715.

Digital Object Identifier 10.1109/TWC.2010.01.081581
TABLE I: Scenarios depending on the terminal constraints.

\begin{tabular}{l|ccc} 
Channel Access & Orthogonal & \multicolumn{2}{c}{ Superposition } \\
\hline Duplexing & - & HD & FD \\
Synchronism & Async. & Sync./Async. & Sync./Async.
\end{tabular}

TABLE II: Low power metrics found in the literature depending on the nodes capabilities. The [*] stands for the cases solved in this paper. The slope $S_{L}$ is obtained with a power allocation linear on the sum power.

\begin{tabular}{l|l|ccc} 
DF and CB & & $\eta$ & $S_{L}$ & $S$ \\
\hline \multirow{2}{*}{ FD } & Async. & {$[5] /[6]$} & {$[6]$} & {$[*]$} \\
\cline { 2 - 5 } & Sync. & {$[5] /[6]$} & {$[*]$} & {$[*]$} \\
\hline OT & Async. & {$[5] /[6]$} & {$[6]$} & {$[*]$} \\
\hline \multirow{2}{*}{ HD } & Async. & {$[*]$} & {$[*]$} & {$[*]$} \\
\cline { 2 - 5 } & Sync. & {$[*]$} & {$[*]$} & {$[*]$}
\end{tabular}

$(S)$ of $\mathrm{R}\left(\frac{E_{b}}{N_{0}}\right)$ at $\frac{E_{b}}{N_{0}}=\frac{E_{b}}{N_{0}}$ min . In [2]-[4] these metrics are used to study the energy efficiency of different communication schemes.

Related Work. The study of the energy efficiency for the RC, see Table II, was initiated in [5] assuming additive white Gaussian noise (AWGN) and constant channels gains. Given that the capacity of the RC is not known, the maximum RPE was found for the capacity lower bound obtained with decode and forward (DF) and the upper bound given by the cut set bound $(\mathrm{CB})$ in [7]. Two scenarios were addressed:

i) A FD relay with simultaneous synchronous transmissions from the source and the relay.

ii) A HD relay with a orthogonal channel access from the source and the relay.

This work was extended in [6] to ergodic fading channels. In this case, the study of the slope was also addressed. The same two scenarios were considered. For the FD case $i$ ), the slope was only computed for asynchronous transmissions. This case was shown to be optimal whenever the channels from the source and/or the relay to the destination do not have a constant component. These works have been extended to other forwarding protocols: the amplify and forward scheme was studied in [8], side-information and linear relaying strategies were studied in [9].

Contributions. We restrict our study to DF and CB bounds on the capacity of the RC with constant channels. Previous results are extended in two main directions, see Table II:

- For the HD case, we study the capacity bounds presented in [10], which improve those studied in [5] and [6], by letting the source and the relay to transmit simultaneously.

- In previous works, the power allocation was restricted to be a linear function of the sum power, also the time intervals were fixed. We remove these constraints. 


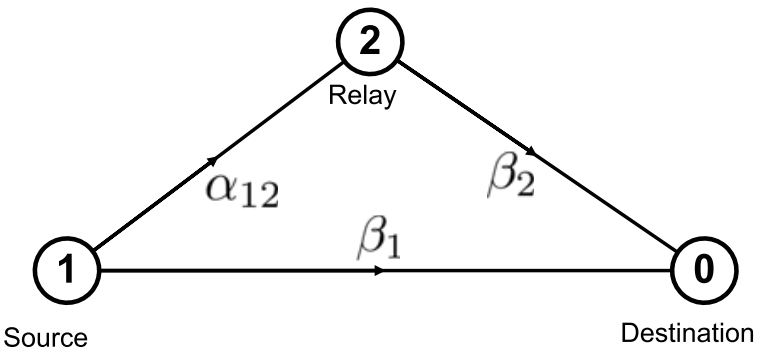

Fig. 1: Relay channel model.

The remainder of the paper is organized as follows. The channel model is presented in Section II. The rate lower bounds with DF and upper bounds with the $\mathrm{CB}$ for relays in HD or FD modes are presented in a unified manner in Section III. In Section IV, the study of the energy efficient regime is formulated and solved for the FD scenario. For the HD scenario, the maximum RPE is studied in Section V and the slope in Section VI. In Section VII, we discuss the gains associated with the different capabilities at terminal. Finally, conclusions are drawn in Section VIII.

\section{Channel MODEL}

Consider the AWGN relay channel depicted in Fig. 1. In HD mode the channel degrees of freedom (time/bandwidth) must be assigned among nodes. Without loss of generality, we consider a fixed bandwidth and assign the transmission time. The transmission period is divided into two intervals. The source transmits to the relay and destination in the first interval $j=1$ while, in the second interval $j=2$, both (HD case) or only the relay (OT case) transmit to the destination. In FD mode, there is only one transmission interval and the index $j$ is dropped. The received signals during the interval $j$ at the relay $Y_{2 j}$ and at the destination $Y_{0 j}$ are given by

$$
\begin{aligned}
& Y_{2 j}=\sqrt{\alpha_{12}} X_{1 j}+Z_{2 j}, \\
& Y_{0 j}=\sqrt{\beta_{1}} X_{1 j}+\sqrt{\beta_{2}} X_{2 j}+Z_{0 j} .
\end{aligned}
$$

We assume, without claim of optimality, that the signals transmitted by the source $X_{1 j}$ and the relay $X_{2 j}$ are Gaussian distributed with zero mean and power $P_{1 j}, P_{2 j}$, respectively. The noise processes at the relay $Z_{2 j}$ and at the destination $Z_{0 j}$ are independent white Gaussian, each one with zero mean and power $N_{0}=1$. The channel gain from the source $l=1$ to the relay $l=2$ is denoted by $\alpha_{12}$, from the source to destination by $\beta_{1}$, and from the relay to destination by $\beta_{2}$. Each transmission uses a fraction $\tau_{j} T$ out of the total transmission period $T$, with $\sum_{j \in\{1,2\}} \tau_{j}=1$. The power allocated to node $l$ during the interval $j$ is denoted as $E_{l j}=\tau_{j} P_{l j}$.

\section{RELAY CHANNEL CAPACITY BOUNDS}

In this work, we consider DF for rate lower bounds and the $\mathrm{CB}$ for rate upper bounds. Depending on the duplexing capability, the transmissions involved are detailed below:

The full duplex relay: The capacity bounds obtained with $\mathrm{CB}$ and DF can be found in $[7 \text {, Theorem } 5]^{1}$. Out of the total

\footnotetext{
${ }^{1}$ The notation used here and the notation used in [7, Theorem 5] are related as $E_{1}^{A}=\rho P_{1}, E_{1}^{S}=(1-\rho) P_{1}$ and $E_{2}=P_{2}$.
}

source power $E_{1}$, a portion $E_{1}^{A}$ is used by the source to inject new information to the channel, using an asynchronous signal $X_{1}^{A}$. The relay assists the communication by transmitting the signal $X_{2}$ with power $E_{2}$. Superposed, if synchronous transmissions are possible, the source transmit coherently with the relay the signal $X_{1}^{S}=\sqrt{\frac{E_{1}^{S}}{E_{2}}} X_{2}$ of power $E_{1}^{S}$. The correlation between the source and relay signal is $\rho=\frac{E_{1}^{S}}{E_{1}}$.

The half duplex relay: The capacity bounds obtained with the CB and DF can be found in $[10]^{2}$. The communication time is divided into two fractions $\tau_{1}$ and $\tau_{2}$. During the first interval, the source dedicates the power $E_{11}=\tau_{1} P_{11}$ to transmit alone, and thus asynchronously, the signal $X_{11}$ with power $P_{11}$. In the second interval, the relay dedicates the power $E_{22}=\tau_{2} P_{22}$ to transmit the signal $X_{22}$ with power $P_{22}$. Meanwhile, the source dedicates the power $E_{12}=E_{12}^{S}+E_{12}^{A}$ to transmit, simultaneously, an asynchronous signal $X_{12}^{A}$ with power $P_{12}^{A}$ and a coherent signal $X_{12}^{S}=\sqrt{P_{12}^{S}} \frac{X_{22}}{\sqrt{P_{22}}}$ with power $P_{12}^{S}$. The correlation between the source and relay transmitted signals is $\rho=\frac{E_{12}^{S}}{E_{12}}=\frac{P_{12}^{S}}{P_{12}}$. If only asynchronous transmissions are possible, then $E_{12}^{S}=0$. In addition, if the channel access between the source and the relay is orthogonal, then $E_{12}^{A}=0$.

The capacity bounds with DF/CB for HD/FD modes and synchronous/asynchronous transmissions can be written in a unified manner as a function of the resource vectors $\tau, \mathbf{E}$ as

$$
R_{F}(\boldsymbol{\tau}, \mathbf{E})=\min _{i \in\{1,2\}} C_{i}(\boldsymbol{\tau}, \mathbf{E})
$$

with

$$
C_{i}(\boldsymbol{\tau}, \mathbf{E})=\sum_{j=1}^{2} \tau_{j} \log \left(1+\frac{g_{j i}(\mathbf{E})}{\tau_{j}}\right) .
$$

Consider $\hat{\alpha}_{12}=\alpha_{12}$ for DF and $\hat{\alpha}_{12}=\alpha_{12}+\beta_{1}$ for CB. For the $\mathrm{FD}$ mode, the power vector is $\mathbf{E}=\left[E_{1}^{A}, E_{1}^{S}, E_{2}\right]$, $\boldsymbol{\tau}=\{1,0\}$ and the functions $g_{j i}, i \in\{1,2\}, j=1$ are given by

$$
\begin{aligned}
& g_{11}(\mathbf{E})=\hat{\alpha}_{12} E_{1}^{A}, \\
& g_{12}(\mathbf{E})=\beta_{1} E_{1}^{A}+\left(\sqrt{\beta_{1} E_{1}^{S}}+\sqrt{\beta_{2} E_{2}}\right)^{2} .
\end{aligned}
$$

For the HD mode, the power vector is $\mathbf{E}=$ $\left[E_{11}, E_{12}^{A}, E_{12}^{S}, E_{22}\right]$, the time-sharing factors are $\boldsymbol{\tau}=\left[\tau_{1}, \tau_{2}\right]$ and the functions $g_{j i} i, j \in\{1,2\}$ are given by

$$
\begin{aligned}
& g_{11}(\mathbf{E})=\hat{\alpha}_{12} E_{11}, \\
& g_{21}(\mathbf{E})=\beta_{1} E_{12}^{A}, \\
& g_{12}(\mathbf{E})=\beta_{1} E_{11}, \\
& g_{22}(\mathbf{E})=\beta_{1} E_{12}^{A}+\left(\sqrt{\beta_{1} E_{12}^{S}}+\sqrt{\beta_{2} E_{22}}\right)^{2} .
\end{aligned}
$$

\section{The ENERGy EFFICIENCY ANALYSIS}

We use the low power analysis developed in [2] to study the spectral efficiency $\mathrm{R}\left(\frac{E_{b}}{N_{0}}\right)$ of the RC in the energy efficient regime. We define the network energy per bit as $E_{b} \triangleq \frac{E}{R(E)}$, where $E$ is the sum power $E=\sum_{l=1}^{2} \sum_{j=1}^{2} E_{l j}$ and $R(E)^{3}$

\footnotetext{
${ }^{2}$ The notation used here and the notation used in [10] are related as $P_{12}^{S}=$ $\rho P_{1}^{(2)}, P_{12}^{A}=(1-\rho) P_{1}^{(2)}$ and $P_{22}=P_{2}$.

${ }^{3}$ The choice of $R$ and $\mathrm{R}$ avoids the abuse of notation that assigns the same symbol to capacity function of $E$ and $\frac{E_{b}}{N_{0}}$.
} 
is the spectral efficiency in bits as a function of $E$. In the low power regime, $\mathrm{R}\left(\frac{E_{b}}{N_{0}}\right)$ is well characterized by computing the minimum energy per bit $\frac{E_{b}}{N_{0}}$ min or equivalently the normalized maximum rate per sum energy as $\eta=\frac{\log _{e} 2}{\frac{E_{b}}{N_{0}} \min }=\dot{R}(0)$ and the slope of $\mathrm{R}\left(\frac{E_{b}}{N_{0}}\right)$ in (bits/s/Hz/3dB) at $\frac{E_{b}}{N_{0} \text { min }}$ as $S=\frac{2[\dot{R}(0)]^{2}}{-\ddot{R}(0)}$, where $\dot{R}(0)$ and $\ddot{R}(0)$ are the first and second order derivative of $R(E)$ in nats at $E=0$. Then, the spectral efficiency can be approximated as [2]

$$
\left.\mathrm{R}\left(\frac{E_{b}}{N_{0}}\right)_{\left.\frac{E_{b}}{N_{0}}\right|_{d B} \rightarrow \frac{E_{b}}{N_{0} \min }}\right|_{d B} \frac{S}{10 \log _{10} 2}\left(\left.\frac{E_{b}}{N_{0}}\right|_{d B}-\left.\frac{E_{b}}{N_{0 \text { min }}}\right|_{d B}\right) .
$$

To compute $\eta$ and $S$, for the RC we need first to obtain the rate $R^{*}(E)$ by maximizing $R_{F}(\boldsymbol{\tau}, \mathbf{E})$ in (2) for all $E$ while fulfilling the resource allocation constraints as

$$
\begin{aligned}
R^{*}(E)=\max _{\boldsymbol{\tau}, \mathbf{E}} \min _{i \in\{1,2\}} C_{i}(\boldsymbol{\tau}, \mathbf{E}) \\
\tau_{1}+\tau_{2}=1, \quad \tau_{1}, \tau_{2} \geq 0, \\
\sum_{\forall j}[\mathbf{E}]_{j}=E, \quad[\mathbf{E}]_{j} \geq 0, \forall j .
\end{aligned}
$$

where $[\mathbf{x}]_{j}$ denotes the $j$-th element in the vector $\mathbf{x}$.

The full duplex relay: In this case, the problem in (7) can be solved in close form as [11]

$$
R^{*}(E)=\log \left(1+\beta_{F D} E\right)
$$

with

$$
\beta_{F D}=\left\{\begin{array}{cl}
\frac{\hat{\alpha}_{12} \hat{\beta}_{2}}{\hat{\alpha}_{12}+\hat{\beta}_{2}-\beta_{1}} & \text { if } \hat{\alpha}_{12}, \hat{\beta}_{2}>\beta_{1}, \\
\beta_{1} & \text { otherwise }
\end{array}\right.
$$

with $\hat{\beta}_{2}=\beta_{2}+\beta_{1}$ for synchronous and $\hat{\beta}_{2}=\beta_{2}$ for asynchronous transmissions. Then, $\dot{R}^{*}(0)=\beta_{F D}, \ddot{R}^{*}(0)=$ $\left(\beta_{F D}\right)^{2}$ and the maximum RPE is $\eta_{F D}=\beta_{F D}$ and the slope is $S_{F D}=2$.

The half duplex relay: In this case, we are unable to find explicitly the pair $(\boldsymbol{\tau}, \mathbf{E})$ that maximizes the rate in (7) for all $E$. Instead, in the following we introduce the notation used in the next sections to compute the first and second order derivatives of $R^{*}(E)$ at $E=0$ :

- $\boldsymbol{\tau}(E)$ and $\mathbf{E}(E)$ are differentiable functions of $E$. At $E=0$, we define $\mathbf{t} \triangleq \boldsymbol{\tau}(0)$, the first order derivatives $\dot{\mathbf{t}} \triangleq \dot{\tau}(0), \dot{\mathbf{e}} \triangleq \dot{\mathbf{E}}(0)$ and the second order derivative $\ddot{\mathrm{e}} \triangleq \dot{\mathbf{E}}(0)$.

- After substituting the resource functions $\boldsymbol{\tau}(E)$ and $\mathbf{E}(E)$ into (2), (3) and (5), we define $R(E) \triangleq$ $R_{F}(\boldsymbol{\tau}(E), \mathbf{E}(E)), C_{E_{i}}(E) \triangleq C_{i}(\boldsymbol{\tau}(E), \mathbf{E}(E))$, and $g_{E_{j i}}(E) \triangleq g_{j i}(\mathbf{E}(E))$.

\section{Maximum Rate Per Energy}

For the HD relay channel, the bounds on the maximum RPE with DF and the CB were first studied in [5] and [6]. The HD limitation was introduced by forcing the channel access from the source and the relay to the destination to be orthogonal or, equivalently, forcing $E_{12}^{A}=0$ and $E_{12}^{S}=0$ in (7). Since simultaneous transmission were not allowed only the asynchronous case was solved. For asynchronous transmissions, no further gains are expected by studying the enhanced bounds in (7), since the maximum RPE bounds obtained using the orthogonal channel access coincide with the ones for a relay in FD mode. For synchronous transmission, we show in the next theorem that the rate bounds in (7) for HD nodes can also attain the bounds on the maximum RPE obtained with a relay in FD mode.

Theorem 1: For the relay channel with synchronous or asynchronous transmissions, the bounds on the maximum RPE using DF for the lower bounds and the $\mathrm{CB}$ for the upper bounds, with a HD relay $\left(\eta_{H D}\right)$ or with a FD relay $\left(\eta_{F D}\right)$ satisfy $\eta_{H D}=\eta_{F D}=\beta_{F D}$ with $\beta_{F D}$ in (9).

Proof: First, $g_{22}(\mathbf{E})$ in (5) can be maximized separately. Let us denote $E^{R}=E_{22}+E_{12}^{S}$, then $g_{22}(\mathbf{E})$ is maximized at $E_{12}^{S}=\frac{\beta_{1}}{\beta_{1}+\beta_{2}} E^{R}, E_{22}=\frac{\beta_{2}}{\beta_{1}+\beta_{2}} E^{R}$ and thus, $g_{22}(\mathbf{E})=\beta_{1} E_{12}^{A}+\hat{\beta}_{2} E^{R}$ with $\hat{\beta}_{2}=\beta_{1}+\beta_{2}$ for synchronous and $\hat{\beta}_{2}=\beta_{2}$ for asynchronous transmissions. Consider, now, the computation of the maximum RPE as $\eta=\dot{R}^{*}(0)$. Employing the notation introduced in the previous section, the rate limits $C_{E_{1}}(E)$ and $C_{E_{2}}(E)$, in order to satisfy the sum and positive power conditions in $(7 \mathrm{c})$, must coincide at $E=0$, namely $C_{E_{1}}(0)=C_{E_{2}}(0)=0$. Nevertheless, each one increases as $\dot{C}_{E_{i}}(0)$. Consequently $\dot{R}(0)$ is found as $\dot{R}(0)=\min _{i \in\{1,2\}} \dot{C}_{E_{i}}(0)$. The derivatives of the rate limits $C_{E_{i}}(E)$ at $E=0$ with $g_{E_{j i}}(E)$ in (5) are given by

$$
\begin{aligned}
& \dot{C}_{E_{1}}(\dot{\mathbf{e}})=\hat{\alpha}_{12} \dot{e}_{11}+\beta_{1} \dot{e}_{12}^{A}, \\
& \dot{C}_{E_{2}}(\dot{\mathbf{e}})=\beta_{1} \dot{e}_{11}+\beta_{1} \dot{e}_{12}^{A}+\hat{\beta}_{2} \dot{e}^{R} .
\end{aligned}
$$

The functions $\dot{C}_{E_{i}}(0)$ depend only on $\dot{\mathbf{e}}$, namely $\dot{C}_{E_{i}}(\dot{\mathbf{e}})$. Consequently, $\dot{R}^{*}(0)$ can be found as

$$
\begin{array}{r}
\dot{R}^{*}(0)=\max _{\dot{\mathbf{e}}} \min _{i \in\{1,2\}} \dot{C}_{E_{i}}(\dot{\mathbf{e}}) \\
\sum_{\forall j}[\dot{\mathbf{e}}]_{j}=1, \quad[\dot{\mathbf{e}}]_{j} \geq 0 \forall j .
\end{array}
$$

The constraints (11b) on $\dot{e}$ are forced by the sum power and positive power constraints in (7c).

Substituting (10) into (11) and solving the resultant problem over $\dot{\mathbf{e}}=\left[\dot{e}_{11}, \dot{e}_{12}^{A}, \dot{e}^{R}\right]$, we obtain: If $\hat{\alpha}_{12} \leq \beta_{1}$ or $\hat{\beta}_{2} \leq \beta_{1}$ only the source transmits, then $\dot{e}_{12}^{A *}=1, \dot{e}_{11}^{*}=0, \dot{e}^{R *}=0$ and $\eta_{H D}=\dot{R}^{*}(0)=\dot{C}_{E_{1}}\left(\dot{\mathbf{e}}^{*}\right)=\dot{C}_{E_{2}}\left(\dot{\mathbf{e}}^{*}\right)=\beta_{1}$. Otherwise, the relay transmits, then $\dot{e}_{11}^{*}=\frac{\hat{\beta}_{2}}{\hat{\alpha}_{12}+\hat{\beta}_{2}-\beta_{1}}, \dot{e}_{12}^{A *}=0, \dot{e}^{R *}=$ $\frac{\hat{\alpha}_{12}-\beta_{1}}{\hat{\alpha}_{12}+\hat{\beta}_{2}-\beta_{1}}$ and $\eta_{H D}=\dot{R}^{*}(0)=\dot{C}_{E_{1}}\left(\dot{\mathbf{e}}^{*}\right)=\dot{C}_{E_{2}}\left(\dot{\mathbf{e}}^{*}\right)=$ $\frac{\hat{\alpha}_{12} \hat{\beta}_{2}}{\hat{\alpha}_{12}+\hat{\beta}_{2}-\beta_{1}}$.

We also provide $\dot{g}_{E_{j i}}^{*}(0)$ for all $i, j$, as we need them to compute the slope in the next section:

\begin{tabular}{llll|l}
$\dot{g}_{E_{11}}^{*}$ & $\dot{g}_{E_{21}}^{*}$ & $\dot{g}_{E_{12}}^{*}$ & $\dot{g}_{E_{22}}^{*}$ & \\
\hline 0 & $\eta$ & 0 & $\eta$ & $\hat{\alpha}_{12}$ or $\hat{\beta}_{2} \leq \beta_{1}$ \\
$\eta$ & 0 & $\frac{\beta_{1}}{\hat{\alpha}_{12}} \eta$ & $\eta \frac{\hat{\alpha}_{12}-\beta_{1}}{\alpha_{12}}$ & otherwise
\end{tabular}

\section{Slope of the SPECTRAl EFFiciency}

The study of the maximum RPE has not revealed the potential gain of FD capabilities at the relays. The same maximum RPE is obtained with a HD relay. Moreover, obtaining the 
maximum RPE is possible even without optimizing the timesharing factors $\tau$ and considering a linear dependence of the power vector on the sum power, i.e. $\mathbf{E}(E)=\dot{\mathbf{e}} E$. Thus, we turn our attention to the analysis of the slope of the spectral efficiency. The study of the slope with a HD relay was first addressed in [6] for ergodic fading channels, assuming orthogonal transmissions. The slope was only obtained for the asynchronous case and the power allocation was restricted to be linear in $E$. For the particular case of constant channels, we solve the synchronous case and also show that the slope can be enhanced by removing the linear power allocation assumption. The next theorem summarizes the obtained results.

Theorem 2: For HD nodes, the slope is given by $S_{H D}=$ 2 if $\hat{\beta}_{2}<\beta_{1}$ or $\hat{\alpha}_{12}<\beta_{1}$ (direct transmission is better), otherwise

$$
S_{H D}=2 \frac{\hat{\beta}_{2}}{\eta}\left(\sqrt{\frac{\hat{\beta}_{2}}{\eta}-1+\left(\frac{\beta_{1}}{\hat{\alpha}_{12}}\right)^{2}}+1-\frac{\beta_{1}}{\hat{\alpha}_{12}}\right)^{-2} .
$$

However, if the power allocation is restricted to be linear in $E$, but the time-sharing vector $\tau$ is optimally assigned, the slope is $S_{H D_{e}}=1+\frac{\beta_{1}}{\hat{\alpha}_{12}}$. Instead, if the power is optimally allocated by the time-sharing factors are fixed to $\tau_{1}=\tau_{2}=\frac{1}{2}$, then

$$
S_{H D_{\tau}}=\left(1+2\left(\frac{\eta}{\hat{\alpha}_{12}}-1\right)\left(\frac{\beta_{1}}{\hat{\alpha}_{12}}\right)\right)^{-1} .
$$

Finally, if, both, the power is restricted to be linear in $E$ and the time-sharing factors are fixed, the slope decreases to $S_{H D_{\tau, e}}=1$. Note that, in all these cases, the maximum RPE is unchanged.

Proof: The slope of the spectral efficiency is computed as $S \triangleq \frac{2\left[\dot{R}^{*}(0)\right]^{2}}{-\ddot{R}^{*}(0)}$. The first-order derivative $\dot{R}^{*}(0)$ was computed in the previous section. After solving (11), both rate limits increase equally, $\dot{R}^{*}(0)=\dot{C}_{E_{1}}(0)=\dot{C}_{E_{2}}(0)$. Thus, $\ddot{R}(0)$ can be computed as $\ddot{R}(0)=\min _{i \in\{1,2\}} \ddot{C}_{E_{i}}(0)$. The second-order derivatives of the rate limits $C_{E_{i}}(E)$ at $E=0$ with $g_{E_{j i}}(E)$ in (5) and $\dot{g}_{E_{j, i}}^{*}(0)$ given in (12) read

$$
\begin{aligned}
\ddot{C}_{E_{1}}(\ddot{\mathbf{e}}, \mathbf{t}) & =-\frac{\left(\dot{g}_{E_{11}}^{*}(0)\right)^{2}}{t_{1}}-\frac{\left(\dot{g}_{E_{21}}^{*}(0)\right)^{2}}{t_{2}} \\
& +\hat{\alpha}_{12} \ddot{e}_{11}+\beta_{1} \ddot{e}_{12}^{A}, \\
\ddot{C}_{E_{2}}(\ddot{\mathbf{e}}, \mathbf{t}) & =-\frac{\left(\dot{g}_{E_{12}}^{*}(0)\right)^{2}}{t_{1}}-\frac{\left(\dot{g}_{E_{22}}^{*}(0)\right)^{2}}{t_{2}} \\
& +\beta_{1} \ddot{e}_{11}+\beta_{1} \ddot{e}_{12}^{A}+\hat{\beta}_{2} \ddot{e}^{R} .
\end{aligned}
$$

The functions $\ddot{C}_{E_{i}}(0)$ depend only on $\ddot{\mathbf{e}}$ and $\mathbf{t}$, namely $\ddot{C}_{E_{i}}(\ddot{\mathbf{e}}, \mathbf{t})$. Then, $\ddot{R}^{*}(0)$ can be bound as

$$
\begin{aligned}
& \ddot{R}^{*}(0)=\max _{\ddot{\mathbf{e}}, \mathbf{t}} \min _{i \in\{1,2\}} \ddot{C}_{E_{i}}(\ddot{\mathbf{e}}, \mathbf{t}) \\
& t_{1}+t_{2}=1, t_{1}, t_{2} \geq 0, \\
& \sum_{\forall j}[\ddot{\mathbf{e}}]_{j}=0, \quad[\ddot{\mathbf{e}}]_{j} \geq 0 \text { if }[\dot{\mathbf{e}}]_{j}=0 \forall j .
\end{aligned}
$$

The constraints on $\mathrm{t}$ in (16b) are forced by the constraints in (7b). The sum power constraint on $\ddot{e}$ in (16c) is forced by the sum power constraint in (7c). Finally, the positive constraint on $\ddot{\mathrm{e}}$ in $(16 \mathrm{c})$ is due to the positive power constraint in (7c). Notice that if $[\dot{\mathbf{e}}]_{j}=0$, then $[\mathbf{E}]_{j}$ satisfies $[\mathbf{E}]_{j}=\frac{[\ddot{\mathbf{e}}]_{j}}{2} E^{2}+o\left(E^{3}\right)^{4}$. Consequently, the positive power constraint $\left([\mathbf{E}]_{j}>0\right)$ forces $[\ddot{\mathbf{e}}]_{j} \geq 0$. To solve (16), we consider two cases:

- if $\hat{\alpha}_{12} \leq \beta_{1}$ or $\hat{\beta}_{2} \leq \beta_{1}$, after substituting $\dot{g}_{E_{j, i}}^{*}(0)$ in (12) and including the sum power constraint as $\ddot{e}_{12}^{A}=$ $-\left(\ddot{e}_{11}+\ddot{e}^{R}\right)$ into (15), we get

$$
\begin{aligned}
& \ddot{C}_{E_{1}}(\ddot{\mathbf{e}}, \mathbf{t})=-\frac{(\eta)^{2}}{t_{2}}-\left(\beta_{1}-\hat{\alpha}_{12}\right) \ddot{e}_{11}-\beta_{1} \ddot{e}^{R}, \\
& \ddot{C}_{E_{2}}(\ddot{\mathbf{e}}, \mathbf{t})=-\frac{(\eta)^{2}}{t_{2}}-\left(\beta_{1}-\hat{\beta}_{2}\right) \ddot{e}^{R}
\end{aligned}
$$

The positive power constraints forces $\ddot{e}_{11}, \ddot{e}^{R} \geq 0$, and $\ddot{C}_{E_{1}}$ and $\ddot{C}_{E_{2}}$ are maximized at $\ddot{e}_{11}^{*}=\ddot{e}^{R *}=0$ and $t_{2}^{*}=$ 1. Then, $\ddot{R}^{*}(0)=\ddot{C}_{1}\left(\ddot{\mathbf{e}}^{*}, \mathbf{t}^{*}\right)=\ddot{C}_{2}\left(\ddot{\mathbf{e}}^{*}, \mathbf{t}^{*}\right)=-(\eta)^{2}$ and $S_{H D}=2$.

- if $\beta_{1} \leq \hat{\alpha}_{12}, \hat{\beta}_{2}$ including the sum power constraint as $\ddot{e}^{R}=-\left(\ddot{e}_{11}+\ddot{e}_{12}^{A}\right)$ into (15), we get

$$
\begin{aligned}
\ddot{C}_{E_{1}}(\ddot{\mathbf{e}}, \mathbf{t}) & =-\frac{\left(\dot{g}_{E_{11}}^{*}(0)\right)^{2}}{t_{1}}-\frac{\left(\dot{g}_{E_{21}}^{*}(0)\right)^{2}}{t_{2}} \\
& +\hat{\alpha}_{12} \ddot{e}_{11}+\beta_{1} \ddot{e}_{12}^{A}, \\
\ddot{C}_{E_{2}}(\ddot{\mathbf{e}}, \mathbf{t}) & =-\frac{\left(\dot{g}_{E_{12}}^{*}(0)\right)^{2}}{t_{1}}-\frac{\left(\dot{g}_{E_{22}}^{*}(0)\right)^{2}}{t_{2}} \\
& -\left(\hat{\beta}_{2}-\beta_{1}\right) \ddot{e}_{11}-\left(\hat{\beta}_{2}-\beta_{1}\right) \ddot{e}_{12}^{A} .
\end{aligned}
$$

First, we assume t constant and maximize $\min \left(\ddot{C}_{E_{1}}, \ddot{C}_{E_{2}}\right)$ over $\ddot{e}_{11}$ and $\ddot{e}_{12}^{A}$. Notice that $\ddot{C}_{E_{1}}$ increases linearly with $\ddot{e}_{11}$ and $\ddot{e}_{12}^{A}$ whereas $\ddot{C}_{E_{2}}$ decreases linearly with both $\ddot{e}_{11}$ and $\ddot{e}_{12}^{A}$. Consequently, $\min \left(\ddot{C}_{E_{1}}, \ddot{C}_{E_{2}}\right)$ is maximized in the equality of (18a) and (18b) at

$$
\begin{aligned}
\ddot{R}^{*}(\mathbf{t}) & =\ddot{C}_{E_{1}}\left(\ddot{\mathbf{e}}^{*}, \mathbf{t}\right)=\ddot{C}_{E_{2}}\left(\ddot{\mathbf{e}}^{*}, \mathbf{t}\right), \\
& =-\frac{\left(\Psi_{1}\right)^{2}}{t_{1}}-\frac{\left(\Psi_{2}\right)^{2}}{t_{2}}
\end{aligned}
$$

with $\left(\Psi_{1}\right)^{2}=\left(1-\frac{\eta}{\hat{\beta}_{2}}\right)\left(\dot{g}_{E_{11}}^{*}(0)\right)^{2}+\frac{\eta}{\hat{\beta}_{2}}\left(\dot{g}_{E_{12}}^{*}(0)\right)^{2}$ and $\left(\Psi_{2}\right)^{2}=\frac{\eta}{\hat{\beta}_{2}}\left(\dot{g}_{E_{22}}^{*}(0)\right)^{2}$. Then, by maximizing (19) over t while fulfilling $t_{1}+t_{2}=1$, we get $\frac{1}{t_{1}^{*}}=1+\frac{\Psi_{2}}{\Psi_{1}}$ and $\frac{1}{t_{2}^{*}}=1+\frac{\Psi_{1}}{\Psi_{2}}$. Notice that since $\left(\Psi_{1}, \Psi_{2} \geq 0\right)$, the positive constraint on $\mathbf{t}^{*}$ in (16b) is always satisfied. Substituting $\mathbf{t}^{*}$ into (19) yields $\ddot{R}^{*}(0)=-\left(\Psi_{1}+\Psi_{2}\right)^{2}$ and the slope is (13).

The suboptimal resource allocations have sense only if the relay cooperates $\left(\beta_{1} \leq \hat{\alpha}_{12}, \hat{\beta}_{2}\right)$ :

i) If the power allocation is restricted to be linear in $E$, then substituting $\ddot{e}_{11}^{*}=\ddot{e}_{12}^{*}=\ddot{e}_{22}^{*}=0$ into (18) and maximizing $\min \left(\ddot{C}_{E_{1}}(\mathbf{0}, \mathbf{t}), \ddot{C}_{E_{1}}(\mathbf{0}, \mathbf{t})\right)$ over $\mathbf{t}$, we obtain $t_{1}^{*}=\frac{\hat{\alpha}_{12}+\beta_{1}}{2 \hat{\alpha}_{12}}, t_{2}^{*}=\frac{\hat{\alpha}_{12}-\beta_{1}}{2 \hat{\alpha}_{12}}$. Notice that since $\hat{\alpha}_{12}>\beta_{1}$, the positive constraint on $\mathbf{t}^{*}$ in (16b) is always satisfied. Then, $\ddot{R}^{*}(0)=\ddot{C}_{E_{1}}\left(\mathbf{0}, \mathbf{t}^{*}\right)=\ddot{C}_{E_{1}}\left(\mathbf{0}, \mathbf{t}^{*}\right)=-\frac{2 \hat{\alpha}_{12}}{\hat{\alpha}_{12}+\beta_{1}}(\eta)^{2}$ and the slope is $S_{H D_{e}}=1+\frac{\beta_{1}}{\hat{\alpha}_{12}}$.

ii) If the time-sharing factors are fixed to $\mathbf{t}_{0}=\left[\frac{1}{2}, \frac{1}{2}\right]$ but $\ddot{\mathrm{e}}$ is optimally assigned. It is direct from (19) that $\stackrel{R}{ }^{*}\left(\mathbf{t}_{0}\right)=$ $-2\left(\Psi_{1}\right)^{2}-2\left(\Psi_{2}\right)^{2}$ and the slope is (14).

${ }^{4} O\left(E^{i}\right)$ is a function that satisfies $\frac{o\left(E^{i}\right)}{E^{i-1}} \underset{E \rightarrow 0}{\rightarrow} 0$. 


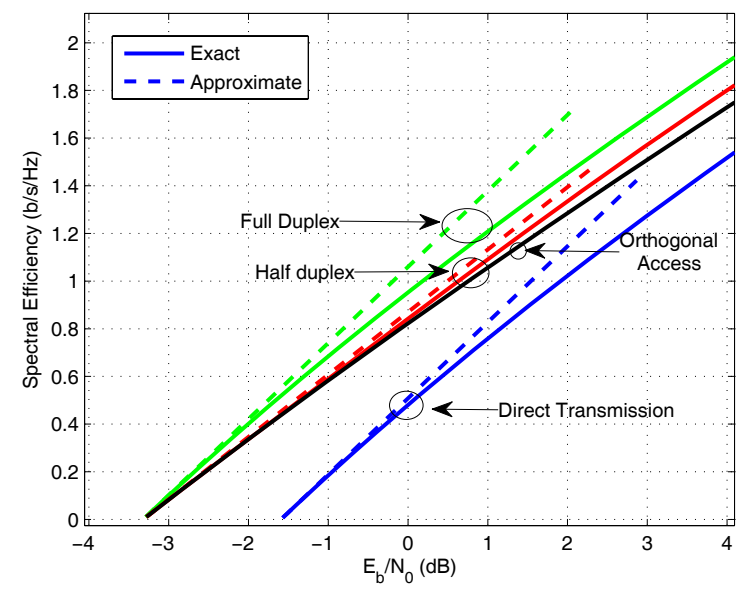

Fig. 2: Relay near the destination at coordinates $(0.75,0.25)$. Approximate and exact rate (bits/s/Hz) versus $\frac{E_{b}}{N_{0}}(\mathrm{~dB})$ for full duplex, half duplex, orthogonal, and direct transmission.

iii) Finally, if $\ddot{\mathbf{e}}=\mathbf{0}$ and $\mathbf{t}=\mathbf{t}_{0}$, we have $\ddot{R}^{*}(0)=$ $\min _{i \in\{1,2\}} \ddot{C}_{E_{i}}\left(\mathbf{0}, \mathbf{t}_{0}\right)=\ddot{C}_{E_{1}}\left(\mathbf{0}, \mathbf{t}_{0}\right)=-2(\eta)^{2}$ and the slope is $S_{H D_{\tau, e}}=1$.

\section{Discussions AND Simulations}

For simulations, we consider distance dependent channel gains with a pathloss exponent $\nu=2$ and $\beta_{1}=1$. The source and the destination are located in a plane at coordinates $(0,0)$ and $(0,1)$, respectively. First, we investigate the gap between the low power approximation and the exact spectral efficiency curve. The spectral efficiency can be approximated as (6), the exact $\mathrm{R}\left(\frac{E_{b}}{N_{0}}\right)$ is here obtained by exhaustive search of the optimal resources allocation. In Fig. 2, we show the spectral efficiency curves for DF with asynchronous transmissions. As pointed out also in [6], if the spectral efficiency is less than $0.3 \mathrm{~b} / \mathrm{s} / \mathrm{Hz}$ the gap between the approximate and exact curves is small in all the studied cases.

Next, we study the impact on the energy efficiency of the terminal capabilities considered:

The channel access. By forcing the source and the relay transmissions to be orthogonal, neither the synchronism nor the full duplex capabilities are used. However, we have seen that if asynchronous and HD transmissions are assumed, then the orthogonal channel access is optimal in terms of both, the maximum RPE and the slope. This result is verified in Fig. 2.

Synchronism. This gain is observed computing the maximum RPE. We have found that this metric is independent of the duplexing capability at the relay. In Fig. 3, we depict the maximum RPE with DF and the $\mathrm{CB}$, for asynchronous and synchronous transmissions as a function of the source to relay position $(d, 0)$. We observe that synchronism provides important gains if the relay is near to the source, even if the relay is farther than the source from the destination (negative axes). However, synchronism provides negligible gains if the relay is near to the destination.

Duplexing. This gain is observed computing the slope of the spectral efficiency $(S)$. This metric determines the bandwidth

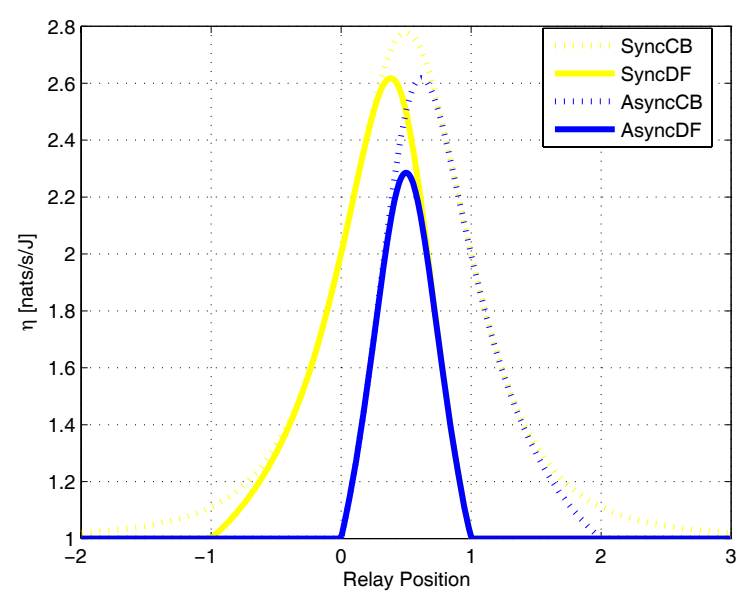

Fig. 3: Maximum RPE versus source to relay distance. The source and the destination are located at $d=0$ and $d=1$ respectively.

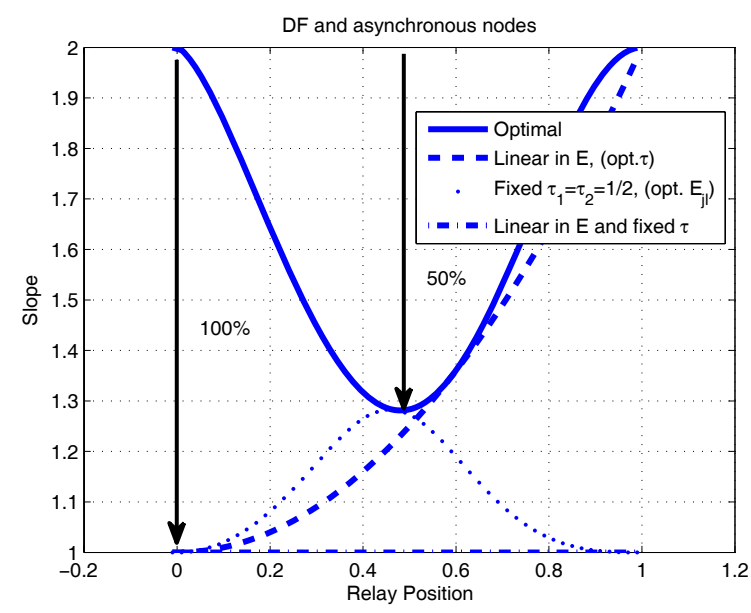

Fig. 4: Maximum slope versus source to relay distance, for different resource allocation assumptions.

efficiency. The required bandwidth for a system to achieve a rate $R$ with power $E$ is $B=\frac{R}{S} \frac{E}{\left.\frac{E}{N_{0} R}\right|_{d B}-\left.\left.\frac{E_{b}}{N_{0}}\right|_{\text {min }}\right|_{d B}}$ [2]. For FD nodes with $\mathrm{DF}$ or the $\mathrm{CB}$, the slope is always $S_{F D}=2$ regardless of the synchronism capability or the relay position. For HD nodes, in Fig. 4 we depict the slope obtained with $\mathrm{DF}$ and asynchronous transmissions as a function of the relay position $(d, 0)$. We can see that, if the relay is near to the middle point between the source and the destination, the HD mode requires approximately $\left(\frac{B_{H D}-B_{F D}}{B_{F D}} \times 100=50 \%\right)$ more of the minimum bandwidth needed with a FD relay for the same rate and transmitted power.

Resource allocation. The performance losses incurred by the suboptimal resource considered in Thm. 2 are studied in terms of the slope in Fig. 4. It is shown that fixing $\tau_{1}=\tau_{2}=\frac{1}{2}$ is a good solution only if the relay is in the middle point between the source and the destination. Likewise, the linear power allocation is a good solution if the relay is near to the destination. However, all the suboptimal resource allocation 
solutions fail if the relay is near to the source. As an example, at $d=0$ the linear power assumption taken in [6] requires $(100 \%)$ more bandwidth.

\section{CONCLUSIONS}

In this work, we studied the energy efficient regime for the relay channel. In particular, we considered the rate bounds given by decode and forward and the cut set upper-bound. The low power analysis tools were applied to investigate the gains provided by synchronism, duplexing and superposition channel access capabilities at the cooperative terminals. We showed that synchronous transmissions are necessary to obtain the maximum RPE but can be achieved with a relay in FD or in HD mode. Furthermore, we showed that for asynchronous transmissions, the orthogonal channel access is sufficient to obtain the maximum RPE. The suboptimality of the orthogonal channel access and the HD mode was determined computing the slope of the spectral efficiency.

\section{REFERENCES}

[1] A. Sendonaris, E. Erkip, and B. Aazhang, "User cooperation diversitypart I: system description," IEEE Trans. Commun., vol. 51, no. 11, pp. 1927-1938, Nov. 2003.
[2] S. Verdú, "Spectral efficiency in the wideband regime," IEEE Trans. Inf. Theory, vol. 48, no. 8, pp. 1319-1343, June 2002.

[3] A. Lozano, A. Tulino, and S. Verdu, "Multiple-antenna capacity in the low-power regime," IEEE Trans. Inf. Theory, vol. 49, no. 10, pp. 25272544, Oct. 2003.

[4] G. Caire, D. Tuninetti, and S. Verdú, "Suboptimality of TDMA in the low power regime," IEEE Trans. Inf. Theory, vol. 50, no. 4, pp. 608-620, Apr. 2004.

[5] A. E. Gamal and S. Zahedi, "Minimum energy communication over a relay channel," in Proc. IEEE Int. Symp. Inf. Theory, Yokohama, Japan, June 2003.

[6] X. Cai, Y. Yao, and G. Giannakis, "Achievable rates in low-power relay links over fading channels," IEEE Trans. Commun., vol. 53, no. 1, pp. 184-194, Jan. 2005.

[7] T. M. Cover and A. E. Gamal, "Capacity theorems for the relay channel," IEEE Trans. Inf. Theory, vol. 25, no. 5, p. 572, Sep. 1979.

[8] Y. Yao, X. Cai, and G. B. Giannakis, "On energy efficiency and optimum resource allocation in wireless relay transmissions," IEEE Trans. Wireless Commun., vol. 4, no. 6, pp. 2917-2927, Nov. 2005.

[9] A. E. Gamal, M. Mohseni, and S. Zahedi, "Bounds on capacity and minimum energy-per-bit for AWGN relay channels." IEEE Trans. Inf. Theory, vol. 52, no. 4, pp. 1545-1561, Apr. 2006.

[10] A. Hst-Madsen and J. Zhang, "Capacity bounds and power allocation for the wireless relay channel," IEEE Trans. Inf. Theory, vol. 51, no. 6, pp. 2020-2040, June 2005.

[11] A. Reznik, S. R. Kulkarni, and S. Verdú, "Degraded Gaussian multirelay channel: capacity and optimal power allocation." IEEE Trans. Inf. Theory, vol. 50, no. 12, pp. 3037-3046, Dec. 2004. 